\title{
Statyba
}

\section{ABOUT METHODS OF THE SUBSTANTIATION OF AN ALLOWABLE RISK LEVEL}

\section{Dr Sc (Eng) A. V. Perelmuter}

To cite this article: Dr Sc (Eng) A. V. Perelmuter (2000) ABOUT METHODS OF THE SUBSTANTIATION OF AN ALLOWABLE RISK LEVEL, Statyba, 6:1, 60-65, DOI:

10.1080/13921525.2000.10531565

To link to this article: https://doi.org/10.1080/13921525.2000.10531565

曲 Published online: 26 Jul 2012.

Submit your article to this journal

III Article views: 35 


\title{
О МЕТОДАХ ОБОСНОВАНИЯ ДОПУСТИМОГО УРОВНЯ РИСКА
}

\author{
А. В. Перельмутер
}

\section{1. Введение}

При решении вопросов о выборе требуемой меры надежности и безопасности строительных объектов (допустимого уровня риска) используются различные, чаще всего неформальные подходы. Bonpoc о возможности назначать этот уровень на основе оптимизационных соображений считается дискуссионным, но дискуссии на эту тему довольно часто ведутся на уровне популистских спекуляций о бесконечной ценности человеческой жизни и т. п. Настоящая работа посвящена некоторым вопросам анализа указанной проблемы и ни в коей мере не претендует на окончательное решение вопроса. Представляется бесспорным важность указания на принщипиальную возможность оптимизационного подхода без уклонения от рассмотрения проблемы по сушеству и без часто используемых ограничений класса рассматриваемых задач так называемыми объектами с чисто экономической ответственностью. Кстати, количество таких систем невелико и полученные для них результаты получили не слишком большое распространение.

\section{2. Особенности оптимизационной постановки} задачи

Для систем с чисто экономической ответственностью обычно минимизируют общие затраты за время эксплуатации объекта T:

$$
C=C_{k}+\int_{0}^{T}\left\{Y_{k}(t) p(t)-B(t)[1-p(t)]\right\} e^{-r t} d t
$$

где $\mathrm{C}_{\mathrm{k}}$ - стоимость конструкшии при ее создании; $Y_{k}(t)$ - убытки от отказа, произошедшего в момент времени $t ; p(t)$ - вероятность отказа в интервале от $t$ до $(\mathrm{t}+\mathrm{dt}) ; \mathrm{B}(\mathrm{t})$ - доход, который приносит объект при безотказной работе; $\mathrm{e}^{-\mathrm{t}}$ - козффициент отдаленности затрат; $r$ - параметр дисконтирования.
Естественно, что при оценке ущерба должны учитываться все компоненты:

- затраты на демонтаж поврежденных конструкций и их замену;

- затраты на восстановление или ремонт;

- потери, связанные с падением деловой активности (упушенная выгода), равные доходу, который могли бы получить за период восстановления работники предприятия;

- компенсашия постороннего ущерба, связанного с нарушением договоров на поставку продукши, штрафами за загрязнение окружаюшей среды и т.п.

При этом иногда обнаруживаются неожиданные факты, заставлягошие пересмотреть привычную систему приоритетов. Достаточно типичным является установленный при анализе проблемы безопасности крупных резервуаров для нефти и нефтепродуктов "закон 1:10:100". Он указывает на то, что убытки от потерянного при разрушении резервуара продукта могут превышать стоимость резервуара в 10 раз, а убытки от загрязнения окружаюшей среды - в 100 раз.

Естественно, что функционал (1) отображает лишь одну из возможных технико-экономических моделей. Они могут быть различными с точки зрения каждой из заинтересованных сторон (владельца объекта, строителя, эксплуатирующей организации, обшства в целом) и по этому поводу часто возникают дискуссии.

Одним из предметов обсуждения является вопрос о дисконтировании затрат, которое реализуется введением множителя $e^{-r}$ в выражение (1) для приведения будущих затрат к начальному моменту времени. Такой способ дисконтирования реализует простое предположение о постоянстве процента с капитала $r_{0}$ (вообще говоря, неверное), так что стоимость $C_{t}$ некоторой суммы денег $C_{0}$, которая будет приобретена или потеряна за $\mathrm{t}$ лет определяется 
известной формулой $\mathrm{C}_{\mathrm{t}}=\mathrm{C}_{\mathrm{o}}\left(1+\mathrm{r}_{\mathrm{o}}\right)^{-\mathrm{L}}=\mathrm{C}_{\mathrm{o}} \mathrm{e}^{-\mathrm{rt}}$, где $r=\ln \left(1+r_{0}\right)$

Дисконтирование затрат учитывает точку зрения владельца капитала, который считает, что любые средства должны приносить доход, а средняя доходность экономической деятельности в обществе равна $r_{0}$, так что заметное отклонение от этого уровня доходности приводит к перетеканию капитала в другой сектор экономики, поскольку имеется возможность разумнее распорядиться капиталовложениями. Дисконтирование «удешевляет» будущие затраты и заставляет дорожить сиюминутными затратами более, чем перспективными доходами и расходами, которые быстро убывают и при относительно небольшом $\mathrm{r}_{\mathrm{o}}=0,08$ уже для $\mathrm{t}=50 \quad \mathrm{C}_{50}=0,018 \mathrm{C}_{\mathrm{o}}$. Однако с точки зрения эксплуатирующей организации, которая, например, арендует объект, нести текущие затраты приходится в полном объеме, а «заработанные» средства чаще всего отсутствуют (истрачены на другие нужды, достались другим лицам и т. п.). В связи с этим зачастую можно услышать жалобы эксплуатационного персонала на непродуманность принятых в свое время решений, недооценку эксптуатационной стадии и т. п.

Для систем с неэкономической ответственностью, отказ которых может быть связан с жертвами или моральным ущербом, проблема существенно усложняется, и по поводу методов ее решения идут многолетние дискуссии. Авторы [1] обрашают внимание на то, что оптимизация по критерию минимизации суммарных затрат $\mathrm{H}_{\text {tot }}$, в которые включена стоимость жизни, приводит к условию:

$$
\Delta \mathrm{H}_{\mathrm{tot}} / \Delta \mathrm{v}=0,
$$

где - $\Delta \mathrm{v}$ количество спасенных жизней при увеличении затрат на $\Delta \mathrm{H}_{\text {tor. }}$ Они утверждают, что это условие можно истолковать следующим образом: сообществу не выгодно платить ни одного доллара за дополнительно спасенную жизнь. Отсюда делается вывод, что оптимизационное решение практически неприемлемо.

Условие (2) можно представить и в других словесных формулировках, звучащих абсолютно не цинично, например, таким образом - «обществу не выгодно платить ни одного доллара за дополнительно спасенную таким образом жизнь, поскольку при других способах использования ограниченных средств (например, за счет организации ранней диагностики рака) можно спасти больше жизней». Однако в пользу оптимизационного подхода в большей мере свидетельствует следующее рассуждение.

Расходы на обеспечение безопасности обычно следуют известному экономическому закону уменьшения отдачи (рис. 1), из которого следует, что первоначально можно снизить относительно большой риск до значительно меньшего уровня при относительно малых затратах, а позже снижение риска обходится все дороже.

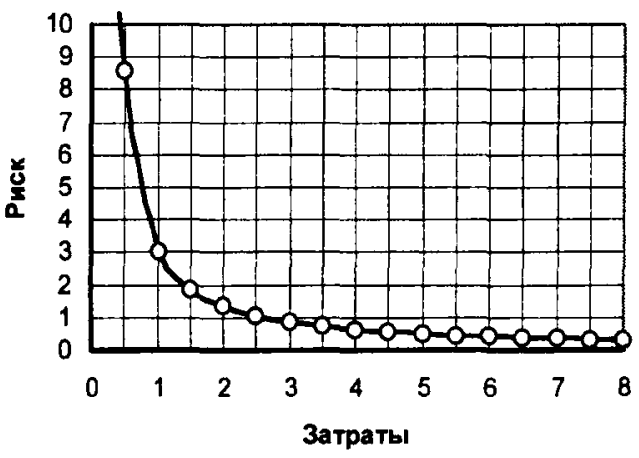

Рис. 1. Уменьшение эффективности затрат

Fig 1. Diminution of efficiency of the costs

Тем не менее создается впечатление, что если не считаться с затратами, то сушествует возможность сократить риск до любого наперед заданного уровня.

Однако сами по себе усилия по снижению риска связаны с определенными действиями (строительными работами, установкой защитного оборудования и др.), при реализации которых также возможны потери здоровья и жизни людей [2]. Об этом свидетельствует статистика несчастных случаев в народном хозяйстве. Например, известно, что каждый миллион тонн добытого на шахтах Донбасса угля «стоит» четырех человеческих жизней. В целом (по данным 1996 года) на Украине производственный травматизм таков, что одна человеческая жизнь связана с выпуском продукции примерно на 11,5 миллионов долларов США.

В связи с этим на графике эффективности затрат появляется дополнительный возрастающй член, который практически не меняет его вид при относительно малых затратах, но реализует минимум на суммарной кривой (рис. 2). Таким образом, проблема имеет решение в оптимизационной постановке. 


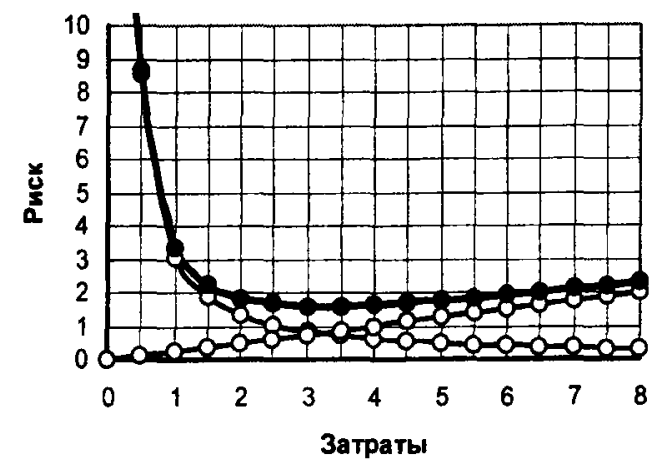

Рис. 2. Зависимость "затраты-риск"

Fig 2. Dependence "costs - risk"

Аналогичная идея была предложена для выработки решений по обеспечению безопасности «саркофага" над аварийным четвертым энергоблоком Чернобыльсхой АЭС. Для различных вариантов восстановления работоспособности и долговечности саркофага оптимизировались обшие «бэрозатраты» с учетом снижения уровня радиационной опасности для населения и возможного радиационного поражения рабочих, осуществляющих ремонт. В некоторых случаях это привело $\mathrm{k}$ нетрадиционным конструктивным решениям.

Важный вывод, который был сделан на основе существования минимума зффективности затрат, состоял в подтверждении того, что может быть обоснована замена ранее применявшегося принципа ALAPA (as low as practicably achievable) - «настолько низко, насколько это практически возможно» новым принщипом ALARA (as low as reasonably achievable) - «настолько низко, насколько это целесообразно». Эта замена применялась и ранее, однако это всегда было связано с острыми дискуссиями, поскольку оставалось неясным, что следует понимать под условием «насколько это целесообразно».

\section{3. Использование эволюционной стратегии}

Детальные решения оптимизационных задач для выбора уровня допустимого риска требуют анализа достаточно больших объемов информации, при этом многие статистические данные оказываются недостаточно достоверными или недоступными. В связи с этим, а также во избежание возможных упреков в цинизме большой популярностью пользуется метод сопоставительного анализа рисков.
Поскольку экономические возможности общества ограничены, представляется разумным следующий подход - общие средства, выделяемые обществом на обеспечение безопасности, должны быть распределены так, чтобы ожидаемое количество возможных жертв по всем видам опасностей было бы минимальным [3]. Это соображение служит основой для сопоставления рисков от различных мероприятий и в различных областях человеческой деятельности.

Несмотря на имеющийся риск, общество продолжает использовать технику, в принципе подверженную отказам, при этом оно приходит к некоторым приемлемым решениям эволюционным путем. Решение принимается на основании интуиции спещиалистов, подкрепленной анализом существующего уровня надежности. Конечно, здесь имеют место постоянные ошибки, однако в общем процессе развития техники происходит своеобразный естественный отбор, в результате которого слишком неправильно спроектированные изделия «вымирают».

Используя эволюционную стратегию, необходимо учитывать не только вероятность возникновения тех или иных ущербных состояний, но и реакцию людей на них. К сожалению, здесь имеются как факты недооценки этой стороны проблемы, так и сугубо конъюнктурные спекуляции на тезисе «общество взволновано».

\section{4. Об отношении к риску}

Каждая крупная техническая авария является событием, которое вызывает определенный общественный резонанс и вырабатывает негативное отношение как специалистов, так и населения $k$ конструкции объекта, с которой произопла авария. Такого рода эффекты значительно увеличиваются при повторных авариях однотипных объектов. Часто бывает достаточным, чтобы после двух-трех аварийных отказов утвердилось прочное мнение об абсолютной ненадежности конструкции некоторого типа. При этом практически игнорируется фактический уровень аварийности. Например, три близких по времени аварии на миллион эксплуатируемых объектов могут вызвать более негативное отношение, чем аналогичная по тяжести последствий единичная авария, приходяшаяся на тысячу эксплуатируемых объектов, хотя реализовавшаяся при этом 
вероятность отказа 1:1000 намного выше, qем 3:1000000.

При использовании метода сопоставления рисков необходимо учитывать некоторые особенности, касаюшиеся формирования общественного мнения. Так, например, многочисленные опросы, проведенные еще до аварии на атомной станции «Три Майл Айленд) и до Чернобыльской катастрофы, показали, что люди считают АЭС очень опасными объектами. При этом реальная угроза атомной энергетики занимала тогда лишь двадцатое место после курения, автомобильных аварий, несчастных случаев при купании и пр. По-видимому, одно из решаюших значений тут имеет разница между добровольным риском и риском по принуждению. Риск собственной жизнью и здоровьем является атрибутом личной свободы, которая высоко ценится, а принуждение к подобному же риску других людей расценивается как покушение на личную свободу, принципиально оцениваемое негативно.

В таблице I приводятся весьма поучительные сравнительные данные по принимаемому и не принимаемому добровольному риску [4].

Второе соображение связано с оценкой числа людей, одновременно подвергающихся риску. Нетрудно проследить, например, по реакции прессы, что одновременная гибель определенного числа людей в авиащионной или морской катастрофе оценивается более остро, чем гибель такого же числа пассажиров, наступившая в результате нескольких автомобильньг катастроф. При этом можно также наблюдать, что большие катастрофы воспринимаются почти одинаково при гибели ста или пятисот человек (эффект насыщения).

Ряд исследований подтвердил, что функциональная зависимость между величиной риска и ожидаемой «выгодой» выражается нелинейной зависимостью, как это показано на рис. 3. Построенная на этом рисунке кривая делит координатную плоскость на две части. Справа от кривой расположены значения, которые могут быть при известных условиях Іриняты, слева располагаются неприемлемые точки.

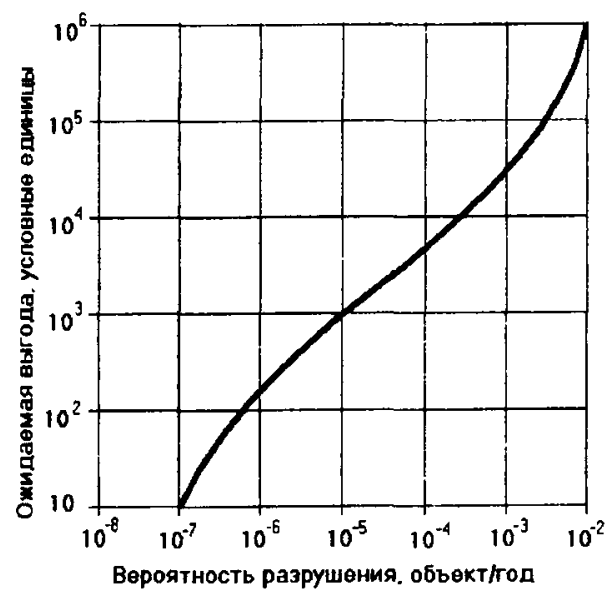

Рис. 3. Нелинейный характер реакции на риск

Fig 3. Non-linear character of a response to risk

Та6лица 1. Риск смертельного исхода в расчете на одного человека в год

Table 1. Risk of fatal outcome for one person per year

\begin{tabular}{|l|c|l|c|}
\hline \multicolumn{2}{|c|}{ Риск, принимаемый добровольно } & \multicolumn{2}{c|}{ Риск, с которым мирятся } \\
\hline Действие & Риск $\left(\times 10^{-5}\right)$ & \multicolumn{1}{c|}{ Действие } & Риск $\left(\times 10^{-7}\right)$ \\
\hline Курение (20 сигарет в день) & 500 & Наезд транспорта & 500 \\
\hline Алкоголь (1 бутылка вина в день) & 75 & Наводнение & 22 \\
\hline Игра в футбол & 4 & Землетрясение & 17 \\
\hline Автогонки & 120 & Торнадо & 22 \\
\hline Вождение автомобиля & 17 & Бури & 8 \\
\hline Вождение мотоцикла & 200 & Молнии & 1 \\
\hline Скалолазание & 4 & Прорыв дамб & 1 \\
\hline Употребление арахисового масла & 4 & Перевозка нефти & 0,2 \\
\hline Противозачаточные таблетки & 2 & Авиакатастрофы & 1 \\
\hline Рентгеновская диагностика & 1 & Радиоактивный выброс АЭС & 1 \\
\hline
\end{tabular}




\section{4. Изменение субъективных оценок во времени}

Предположим, что передача информации о произошедшей аварии от лиц, осведомленных о таком событии, $\mathbf{k}$ неосведомленным лицам происходит примерно с постоянной интенсивностью. При этом чем шире круг осведомленных, тем большему числу лиц могут быть переданы сведения об аварии. Однако с течением времени распространение сведений начинает замедляться, так как иссякает круг неосведомленньгх слушателей.

Таким образом, скорость прироста числа осведомленных лиц С может быть представлена как

$$
\frac{\mathrm{dC}}{\mathrm{dt}}=\alpha \mathrm{C}(1-\mathrm{C} / \mathrm{C})
$$

где С - общее количество потенциальных слушателей, т. е. число лиц, для которых сведения об аварии небезразличны, а $\alpha$ - константа, характеризующая интенсивность распространения сведений.

Решением дифференциального уравнения (3) является известная логистическая кривая, которая описывает рост большинства популяций (в данном случае это популяция осведомленных лиц), и может быть представлена в виде:

$$
C=C\left\{1+\left(\frac{C}{C_{0}}\right) \exp \left[-\alpha\left(t-t_{0}\right)\right]\right\}^{-1},
$$

где через $\tilde{\mathrm{N}}_{0}$ обозначено число лиц, осведомленных об аварии с начального момента времени $\mathrm{t}_{0}$. B дальнейшем для простоты записи будем считать $t_{0}=0$.

Реакция человека на любую новость со временем теряет свою остроту и можно предположить существование зависимости:

$$
R(\tau)=R_{0} \exp [-\beta(\tau-t)]
$$

для описания остроты реакции человека на известие об аварии, полученное им в момент времени t. Здесь через $\beta$ обозначен параметр «скорости успокоения) человека.

Таким образом, группа людей в количестве $\mathrm{dC}(\mathrm{t})$, узнавшая об аварии в момент времени $t, k$ моменту $\tau$ будет иметь остроту восприятия события:

$$
\mathrm{dC} \cdot R(\tau)=A \cdot \exp (-\beta \mathrm{t})\left[1+\left(C / C_{0}-1\right) \exp (-\alpha t)\right]^{-2},(6)
$$

где введено обозначение

$$
A=\alpha R_{0} \mathcal{Q}\left(\frac{C}{C_{0}}-1\right) \cdot \exp (-\beta \tau)
$$

для множителя, не зависяшего от $\mathrm{t}$.

Выражение для суммарной реакция общества $U$ на момент времени $\tau$ можно получить, интегрируя (6). Оно будет иметь вид:

$$
U(\tau)=A \int_{0}^{\tau} \exp (-\beta t)\left[1+\left(C / C_{0}-1\right) \exp (-\alpha t)\right]^{-2} d t .
$$

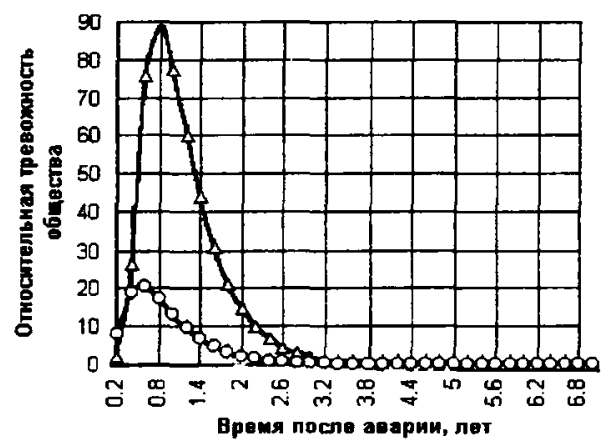

Рис. 4. Изменение уровня тревожности

Fig 4. Modification of a level of uneasiness

На рис. 4 представлены характерные кривые относительной величины $\mathrm{U}(\tau) / \mathrm{CR}_{0}$, вычисленные при $\beta / \alpha=0,5$ и двух значениях безразмерного параметра $\sigma=\tilde{\mathbf{N}}_{0} / \mathrm{C}$ (кружки для $\sigma=0,01$, треугольники для $\sigma=0,1)$. Видно, как первоначальное нарастание относительной тревожности сменяется ее падением вследствие потери остроты восприятия.

Очевидно, что чем больше масштаб аварии $\mathbf{M}$, тем большее число лиц оповещается о ней и, следовательно, можно предполагать существование зависимости типа:

$$
\sigma=c_{0} M
$$

где параметр $c_{0}$ в первом приближении можно оценить, вычисляя круг лиц, которым сообщение об аварии должно поступать немедленно.

Дия параметра $R_{0}$ следует учитывать упомянутый ранее эффект насыщения, когда реакция на масштаб аварии возрастает нелинейно и затухает по мере роста $\mathrm{M}$. Такого рода психологическая реакция давно привлекла внимание в теории полезности применительно к азартным играм (разумеется, что в нашем случае следует говорить об «отрицательной 
полезности»). Часто принимается, что полезность приращения интереса $\mathrm{dX}$ (например, к выигрышу) прямо пропорциональна $\mathrm{dX}$ и обратно пропорциональна X. Это равносильно тому, что сам интерес пропорционален логарифму, т. е. можно принять

$$
R_{0}=\ln \left(r_{0} M\right)
$$

Эта зависимость, которая в отличие от математического ожидания является «психологическим ожиданием», при подходящем подборе параметра $r_{0}$ позволяет учесть наблюдаемые особенности поведения людей при получении известия об аварии.

\section{5. Выводы}

Приведенные математические модели и соображения не противоречат наблюдаемым явлениям и могут использоваться при разработке нормативов уровня допустимого риска. Для их практинеского использования потребуется провести сопоставительные расчеты по оценке параметров математической модели с нспользованием различных указанных подходов. Сопоставление следует выполнить с фактически достигнутым в различных отраслях техники уровнем безопасности, для чего в настоящее время предпринято специальное изучение отчетных данных об аварийности.

\section{Литература}

1. Г. Аугусти, А. Барата, Ф. Кашиати. Вероятностные методы В строительном проектировании. М. Стройиздат, $1988.584 \mathrm{c}$.

2. С. К. Блэк, Ф. Нихаус. Насколько безопасно «слишком» безопасное? // Бюллетень МАГАТЭ. Книга 22, № 1, c. 47-58.

3. Б. И. Снарскис. Основы теории заласов несушей способности строительньтх конструкций: Автореф. дис. ... д-ра техн. наук / ЦНИИСК им. Кучеренко. М., 1969. $35 \mathrm{c}$

4. Т. А. Клетц. Выгоды и риск: сравнительная оценка в связи с потребностями человека // Бюллетень МАГАТЭ. Книга 22, № 5/6, с. 2-14.

5. А. В. Перельмутер. Избранные проблемы належности и безопасности строительньг конструкций. Киев: Изд. Укрниипроектстальконструкция, 1999. 210 с.

Iteikta 19990815

\section{APIE LEISTINO RIZIKOS LYGIO PAGRINDIMO METODUS}

\section{A. V. Perelmuter}

\section{Santrauka}

Analizuojama leistino rizikos lygio optimalaus parinkimo užduotis, ypaž tais atvejais, kai galimos žmoniu aukos. Lyginami skirtingi požiūriai i žmogaus gyvybès kainos problemą nagrinejamas požiūris, kuris remiasi išgelbèty gyvybił skaiciaus palyginimu su ta rizika, kuri tenka žmonems, atliekantiems gelbejimo darbus. Pateikiama informacija apie realios rizikos ivairiose żmoniu veiklos srityse lygi. Aprašomas visuomenès nuomonès formavimo avarijos atveju matematinis modelis ir analizuojami visuomenès reakcijos pokyčiai laikui bėgant. Aprašomi efektai, šiaip jau ignoruojami vertinant rizika, taip pat avarijos mastelio efektas, nurodoma nelinijinè nelaimingo atsitikimo masto priklausomybe nuo visuomenès reakcijos.

\section{ABOUT METHODS OF THE SUBSTANTIATION OF AN ALLOWABLE RISK LEVEL}

\section{A. V. Perelmuter}

Summary

The problem of admissible risk optimisation is described in detail, especially for the case of possible human victims. Different points of view on the problem of shuman being cost» are compared, and the approach based on comparing the numbers of saved lives and the risk for the persons, providing the life saving are considered. An information on actual risk level for different kinds of human activities is presented. The mathematical model of creating the public opinion in case of accidents and analysis of changing the function of society discomposibility function in time are presented. Effects commonly ignored during risk estimation are analysed - like departure from the principle of equal providing the risks for objects of equal responsibility, but different quantity and presence of non-linear dependence between the severity of accidents and public reaction.

Anatoly V. PERELMUTER. Dr Sc (Eng), Professor. Head of laboratory at Ukrainian Institute of Steel Construction and Chief Researcher at the Environmental and Resource Research Institute of National Security and Defence Council of Ukraine. 13, Chokolovsky bld., room 522, Kiev, 252680, Ukraine, Email: scad@erriu.ukrpack.net.

Member of Ukrainian Academy of Civil Engineering, member of National Committee of Theoretical and Applied Mechanics. Author and co-author of 7 monographs and more than 150 scientific articles. Research interests: non-linear problems in structural mechanics, reliability theory, steel constructions, computer-aided analysis. 